\title{
Sexual dimorphic responses of C57BL/6 mice to Fisetin or Dasatinib and Quercetin cocktail oral treatment
}

Yimin Fang ${ }^{1}$, David Medina ${ }^{2}$, Robert Stockwell ${ }^{2}$, Sam McFadden ${ }^{1}$, Kathleen Quinn ${ }^{1}$, Mackenzie

R. Peck ${ }^{1}$, Andrzej Bartke ${ }^{2,3}$, Kevin N. Hascup ${ }^{1,3,4}$, Erin R. Hascup ${ }^{1,4, *}$

${ }^{1}$ Department of Neurology, Dale and Deborah Smith Center for Alzheimer's Research and Treatment, Neuroscience Institute, Southern Illinois University School of Medicine, Springfield, IL 62702, USA.

${ }^{2}$ Department of Internal Medicine, Southern Illinois University School of Medicine, Springfield, IL 62702, USA.

${ }^{3}$ Department of Medical Microbiology, Immunology and Cell Biology, Southern Illinois University School of Medicine, Springfield, IL 62702, USA.

${ }^{4}$ Department of Pharmacology, Southern Illinois University School of Medicine, Springfield, IL 62702, USA.

*Corresponding Author: Erin R. Hascup, Department of Neurology, Dale and Deborah Smith Center for Alzheimer's Research and Treatment, Southern Illinois University School of Medicine, P.O. Box 19628, Springfield, IL 62794-6928, USA Tel: 217-545-6988, Email: ehascup@siumed.edu

Key words: senolytic drugs, cognition, adiponectin, glucose metabolism 


\begin{abstract}
Senolytic treatment in aged mice clears senescent cell burden leading to functional improvements. We hypothesized that administering senotherapeutics in young adulthood of mice would slow physiological markers of aging through mid-life. C57BL/6 mice were treated monthly with either Fisetin or a Dasatinib (D) plus Quercetin (Q) cocktail from 4-13 months of age. Fisetin treated male mice had reduced senescence-associated secretory phenotype (SASP), enhanced glucose and energy metabolism, improved cognitive performance, and increased hippocampal expression of adiponectin 1 receptor and glucose transporter 4 . D+Q treated females had increased SASP expression along with accumulation of white adipose tissue, reduced energy metabolism, and cognitive performance. Senotherapeutics in young adulthood, has beneficial, negligible, or detrimental effects in mice dependent upon sex and treatment.
\end{abstract}




\section{Introduction}

Cell senescence is a phenomenon defined as an irreversible cell cycle arrest, and is associated with the fundamental aging processes and age-related diseases ${ }^{1}$. Although this process is essential for preventing replication of damaged DNA thereby suppressing tumor formation for cancers, accumulation of senescent cells with aging produces senescenceassociated secretory phenotype (SASP) ${ }^{1,2}$. SASP includes chronic inflammation ${ }^{3,4}$, DNA damage $^{5,6}$, mitochondrial dysfunction ${ }^{7}$, immune cell dysfunction ${ }^{8}$, ROS generation ${ }^{5,9}$, and brain protein aggregation ${ }^{10,11}$, all of which may be factors that predispose individuals to a multitude of age-related disorders ${ }^{1}$.

Fisetin and quercetin (Q) are plant-derived flavonoids that offer cytoprotection against cellular stress and act as anti-inflammatory, chemopreventive, chemotherapeutic, and senotherapeutic agents ${ }^{12,13}$. Additionally, Q incites immune response against allergic diseases ${ }^{13,14}$. Dasatinib (D) is a tyrosine kinase inhibitor used to treat leukemia ${ }^{15}$ and is routinely used in combination with $\mathrm{Q}$ to improve the senotherapeutic potency. Fisetin and D+Q selectively clear senescent cells ${ }^{16}$, thereby delaying aging-associated disorders and improving healthspan and lifespan. This has been observed after reducing senescent cell burden in progeroid mice or in 2224 month old C57BL/6 mice ${ }^{17-19}$. Moreover, deletion of senescent cells from the brain genetically or pharmacologically with senolytic drugs led to functional improvements in mouse models of neurodegenerative diseases such as Parkinson's and Alzheimer's disease ${ }^{10,20-22}$.

These studies have shown senolytics can reduce senescent cell burden and have positive impacts on animals with accelerated aging, advanced age, or neurodegenerative disorders. Accordingly, senotherapeutics are currently marketed as anti-aging therapies where young, healthy adults can take these products as dietary supplements. 
However, less is known regarding their anti-aging effects of these compounds when administered prior to significant senescent cell accumulation. Thus, the experiments were designed to examine the long-term effects of monthly oral treatment with Fisetin or a D+Q cocktail when administered to C57BL/6 mice starting at 4 months age. Since aging alters numerous biological functions, we examined morphological, metabolic, and cognitive components that are known to be affected by senescent cell accumulation. The results presented here indicate that monthly administration of Fisetin or D+Q had sexually dimorphic effects which also depended on treatment type in $\mathrm{C} 57 \mathrm{BL} / 6$ mice. We highlight a potential new senotherapeutic mechanism of action involving the beneficial roles of glucose and adiponectin signaling both peripherally and centrally.

\section{Results}

\section{Senolytic treatment altered the SASP profile of C57BL/6 mice in a sex dependent manner.}

It has been reported that senolytic drug treatment decreased senescent burden in 22-24 month old C57BL/6 mice leading to functional improvements ${ }^{17}$. However, it was unknown whether the same treatment administrated monthly starting at a younger age (4 months old) would slow physiological markers of aging. To examine this, Fisetin or a cocktail of D+Q were orally administered in C57BL/6 mice at 4 months of age and continued once each month for 9 months. All mice underwent glucose tolerance test (GTT) and insulin tolerance test (ITT) to evaluate glucose homeostasis after five treatments at 9 months of age. Indirect calorimetry to determine energy metabolism, and Morris water maze (MWM) and novel object recognition (NOR) to ascertain cognitive abilities were performed after eight treatments at the age of 12 
months. At approximately 13 months of age, the mice were sacrificed to determine body composition and collect plasma and tissues for analysis of SASP markers. The experimental paradigm is shown in Fig 1a.

To investigate whether the compounds used in this study decreased senescent cell burden, expression profiles contributing to SASP and other senescent markers were measured in gonadal adipose depots by RT-PCR. To evaluate aging effects, additional groups of untreated young (four months of age) and old (twenty-one months of age) mice were included. The results showed that in both the male and female mice, the transcriptional levels of $\mathrm{p} 16^{\text {Ink } 4 \alpha}$ (Fig. $\left.1 \mathrm{~b}\right)$ and p21 ${ }^{\text {Cip } 1}$ (Fig. 1c) and pro-inflammatory markers associated with senescence, including TNF $\alpha$ (Fig. 1d), MCP 1 (Fig. 1e), IL-6 (Fig. 1f) and IL-10 (Fig. 1g), were lower in the young nontreated C57BL/6 mice than in the aged non-treated mice. The vehicle treated littermate control animals sacrificed at 13 months of age had similar levels of the above mentioned SASP markers as the untreated 21 month old mice (Fig. 1b-1g). Interestingly, the effects of senolytic drugs were sex-dependent. The treatment with Fisetin or $\mathrm{D}+\mathrm{Q}$ reduced gene expression levels of $\mathrm{p} 16^{\mathrm{Ink} 4 \alpha}$ in the male mice, but had no effects in the female mice (Fig. 1b). The treatment with Fisetin reduced the levels of $\mathrm{p} 21^{\mathrm{Cip} 1}, \mathrm{TNF} \alpha$, and IL-6 in the male mice, but had no effect in the female mice (Figs. 1c, 1d, \& 1f), while the treatment with D+Q raised the levels of p2 ${ }^{\text {Cip} 1}$, MCP1, IL-6, and IL-10 (Figs. 1c \& 1e-1g) in the female mice. Together, the data showed that Fisetin treatment reduced SASP and senescent cell burden in male C57BL/6 mice but had no effects in female mice. The D+Q treatment increased, rather than decreased, SASP and senescent cell burden in female C57BL/6 mice, while showing limited efficacy in male mice. 


\section{The senolytic treatment led to increased body weight and adiposity in female C57BL/6 mice that corresponded with increased markers of lipid synthesis and down-regulation of} thermogenesis.

Given the observation that C57BL/6 mice responded to the senolytic treatment in a sexually dimorphic manner, it was important to know how the physiological parameters including body composition, glucose utilization, and energy metabolism were affected by treatment. The results showed that body weight in the male mice was not changed by either treatments, while it was increased in the female mice by monthly D+Q administration (Fig. 2a). Body composition analysis indicated that the increased body weight of $\mathrm{D}+\mathrm{Q}$ treated female C57BL/6 mice was attributed to an elevation of adiposity (Fig. 2b) including subcutaneous (Fig. 2c) and gonadal (Fig. 2d) white adipose tissue (WAT) depots. No changes were observed in the perirenal WAT depot weight (Fig. 2e) nor the interscapular brown adipose tissue (BAT) (Fig. 2f). No changes in body weight or composition were observed after nine months of Fisetin treatment in female mice (Figs 2a-f), and no changes in these parameters were detected in male mice receiving either of the senolytic treatments.

The accumulation of WAT in D+Q treated female mice could result from upregulated lipid deposition in WAT, or downregulated thermogenic activity in BAT. Thus, the expressions of genes related to lipid metabolism in gonadal WAT, and to uncoupling of the mitochondrial electron transport chain in BAT were examined. The results showed that Acetyl-CoA carboxylase (ACC), the rate-limiting enzyme for the lipid synthesis pathway, was up-regulated in $\mathrm{D}+\mathrm{Q}$ treated female mice (Fig. 2g). In contrast to WAT, uncoupling protein 1 (UCP1), a key regulator of non-shivering thermogenesis, (Fig. 2h) and its upstream activator PPAR $\gamma$ (Fig. 2i) 23,24 were downregulated in $\mathrm{D}+\mathrm{Q}$ treated female mice. The data from WAT and BAT support that 
$\mathrm{D}+\mathrm{Q}$ treated female mice had higher lipid synthesis activity, mediated by increased ACC, and lower thermogenic activity, via reduced UCP1 and PPAR $\gamma$. These changes could cause WAT accumulation subsequently leading to the observed SASP increases shown in Fig. 1.

\section{Fisetin treatment improved glucose metabolism and increased plasma adiponectin in male C57BL/6 mice.}

Considering adipocytes are involved in glucose metabolism, we wanted to determine treatment effects on peripheral glucose utilization. The results showed that Fisetin treatment improved glucose clearance as measured by GTT (Fig. 3a \& 3c) in male mice. Although D+Q treated female mice accumulated more adipose tissues (Fig. 2b), they did not have impaired glucose metabolism (Fig. 3b \& 3c). No differences in insulin sensitivity between treated and littermate control mice were observed as determined with ITT (Fig. 3d-3f). Additionally, plasma insulin levels (Fig. 3g) were similar across treatments in both the female and male mice. Interestingly, plasma adiponectin (an adipokine that is involved in regulating glucose levels) was significantly increased in the senolytic treated male mice but not in the treated female mice (Fig. 3h). The data indicated that monthly Fisetin treatment improved peripheral glucose metabolism in the male mice with increased adiponectin levels and enhanced glucose clearance. $\mathrm{D}+\mathrm{Q}$ treatment did not affect glucose metabolism despite this senolytic treatment causing WAT accumulation in the female mice.

Fisetin treatment enhanced energy metabolism in male C57BL/6 mice, while D+Q treatment reduced energy metabolism in female $\mathrm{C57} \mathrm{BL} / 6$ mice. 
Energy metabolism is strongly associated with aging and is a contributing factor to adipose accumulation ${ }^{25,26}$. In the present study, the effects of senolytic treatment on energy metabolism were assessed by indirect calorimetry. Oxygen consumption $\left(\mathrm{VO}_{2}\right)$ (Fig. 4a) and energy expenditure (EE) (Fig. 4c) were increased after Fisetin treatment in male mice, while both parameters were decreased by $\mathrm{D}+\mathrm{Q}$ treatment in female mice (Fig. $4 \mathrm{~b} \& 4 \mathrm{~d}$ ). Thus, Fisetin enhanced energy metabolism in male mice, while $\mathrm{D}+\mathrm{Q}$ reduced energy metabolism in the female mice, potentially contributing to WAT accumulation. Respiratory quotient (RQ) is the ratio of carbon dioxide produced and oxygen consumed by the body, reflecting substrate utilization for energy generation ${ }^{27}$. In the current study, RQ for Fisetin treated male mice was reduced indicating a shift in metabolic substrate utilization from mixed lipids and carbohydrates $(\mathrm{RQ} \sim 0.8)$ to predominantly lipids $(\mathrm{RQ} \sim 0.7)^{27}$ (Fig. 4e). The RQ of female mice treated with $\mathrm{D}+\mathrm{Q}$ did not change (Fig. 4f). In summary, monthly treatment of male mice with Fisetin increased energy metabolism as indicated by enhanced $\mathrm{VO}_{2}$ and $\mathrm{EE}$ and shifted substrate utilization to predominantly lipids. In contrast $\mathrm{D}+\mathrm{Q}$ treatment decreased energy metabolism as indicated by reduced $\mathrm{VO}_{2}$ and $\mathrm{EE}$ without a concomitant change in substrate utilization.

\section{Fisetin treatment improved spatial cognitive performance in male C57BL/6 mice, while D+Q treatment impaired object recognition in female C57BL/6 mice.}

Although deletion of senescent cells by the senolytic compounds led to functional improvements in models of neurodegenerative diseases ${ }^{20,21}$, it remains to be elucidated whether the monthly senolytic treatment could affect cognitive performance in normal aging mice. To 
investigate the effects of senolytic treatment on cognitive functions, the MWM spatial learning and memory paradigm and a NOR task were performed. The results from MWM indicated that over 5 training sessions, the path efficiency was increased (Fig. 5a), and the corrected integrated path length (CIPL) (Fig. 5b) was reduced significantly in the Fisetin-treated male mice indicative of improved learning. Neither Fisetin nor D+Q treatment affected MWM learning in the female mice (Fig 5c \& 5d). Platform entries during the delayed probe challenge was not affected by either treatment in both sexes (Fig. 5e) suggesting spatial memory recall was unaffected. The results of NOR showed that the exploration preference for the novel object measured by retention index was decreased in the $\mathrm{D}+\mathrm{Q}$ treated female mice (Fig. 5f), indicating reduced memory recall in this group. Considering the effects of senolytic compounds on cognitive performance, several genes related to synaptic plasticity ${ }^{28}$ were examined by RT-PCR in the hippocampus. Among twelve genes tested (Fig. 6a-d), the expression of glucose transporter 4 (Glut4) and adiponectin receptor 1 (AdipoR1) (Fig. 6b) were upregulated in Fisetin treated male mice. The expression of the rest of the examined genes was not affected by Fisetin, and we did not detect any effects of D+ Q treatment on hippocampal gene expression. These results indicate that adiponectin signaling and glucose transport may have been enhanced in the brain of Fisetin treated male mice. Interestingly, this is consistent with improved peripheral glucose metabolism in these animals (Fig. 3a-c).

\section{Discussions and conclusions}

Administration of senolytic compounds is a novel approach to removing senescent cell accumulation and thereby reducing inflammation. These compounds are being investigated as potential lifespan and healthspan extending therapies and may also have 
applications in the treatment of numerous metabolic and neurodegenerative disorders ${ }^{10,20-22}$. Since Fisetin and Q are naturally occurring plant flavonoids, they are readily available as dietary supplements for use without a prescription. There is little information on the efficacy of these compounds when taken at young ages and for prolonged periods of time. The data presented here indicates that these compounds have sexually dimorphic effects, each with health benefits and risks. The monthly dosing strategy used in this study was based on previous publications showing that markers of cell senescence are still reduced after four week off-treatment period ${ }^{1}$. Prior research suggests that senotherapeutic compounds work either by clearing senescent cells or reversing cellular senescence rather than preventing cells from entering a senescent state. Since maintaining an effective concentration dosage is evidently not required, intermittent dosing may be preferable by avoiding the development of drug tolerance and thus providing therapeutic efficacy over a longer duration.

Monthly Fisetin treatment was efficacious in male C57BL/6 mice when started at 4 months of age. This dosing strategy had little to no effect in the female mice when compared to vehicle treatment. Nine months of Fisetin treatment efficiently reduced SASP in WAT of 13 month old male mice as lower expression of $\mathrm{p} 16^{\mathrm{Ink} 4 \alpha}, \mathrm{p} 21^{\mathrm{Cip} 1}, \mathrm{TNF} \alpha$, and IL-6 were observed, indicating reduced senescent cell burden (Fig. 1). After five Fisetin doses, 9 month old male mice had improved glucose metabolism as supported by better glucose clearance (Fig 3a \& 3c) and elevated plasma adiponectin (Fig. 3h). Improved energy metabolism was also supported by increased $\mathrm{VO}_{2}$ and $\mathrm{EE}$ (Fig. 4a \& 4c) along with reduced RQ (Fig. 4e).

In the present study, D+Q treatment showed no efficacy in male C57BL/6 mice and was detrimental to the female mice. In females, senescent markers and pro-inflammatory 
cytokines were increased (Fig. 1) attributed to larger WAT depots (Fig 2c \& 2d). The D+Q treated female mice gained body weight mainly from this WAT accumulation (Fig. 2). We suspect that increased ACC (the rate-limiting enzyme in lipid synthesis) in WAT and reduced UCP1 and PPAR $\gamma$ activity in BAT (Fig. 2g-i), and reduced energy metabolism (Fig. 3) in D+Q treated female mice have all contributed to the increased abdominal adiposity (Fig. 2). Because obesity can lead to adipose tissue entering a senescent-like state at a young age $^{29}$, the accumulation of abdominal WAT in D+Q treated female mice could result in increased senescent cell burden. This possibility is supported by expression of SASP-related genes in D+Q treated females resembling or even exceeding the values measured in much older (21 month old) untreated females (Fig. 1). The reasons for the detrimental outcome of $\mathrm{D}+\mathrm{Q}$ treatment in the female mice remain to be elucidated. A recent publication reported similar observations in a C57BL/6 mouse model of hepatocellular carcinoma (Raffaele M et al., 2021). However, our data suggest that the age when senolytic treatment is initiated may play an important role regarding therapeutic outcome. Additionally, the compound tolerance rate in female mice might be faster than in males indicating the need for a longer duration between treatments.

In C57BL/6 mice, cell senescence usually becomes prominent by approximately 14 months of age ${ }^{1}$, and treatment with senolytic drugs at this or a later ages cleared senescent cells and led to various beneficial effects ${ }^{17,18}$. However, it was unclear whether chronic treatment with senolytic drugs started at an earlier age (prior to reported senescent cell accumulation) in C57BL/6 mice would have similar effects as the reported observations during later age ${ }^{17,18}$. This gap in knowledge is particularly relevant given that Fisetin and Q 
are available without a prescription and may be taken by younger adults. Our data revealed that such treatment indeed had impacts on the mice as they reached median lifespan. However, the effects were sexually dimorphic. C57BL/6 male, not female, mice receiving Fisetin treatment had beneficial responses mediated through reduced SASP and improved glucose and energy metabolism. Female, not male, mice receiving a D+Q cocktail treatment had detrimental responses with reduced energy metabolism, increased WAT accumulation, and elevated SASP.

Moreover, Fisetin-treated male mice had not only experienced improvements in the measured markers of peripheral health, but also enhanced their spatial learning during the MWM training sessions (Fig 5 a and 5b). Although we did not observe changes in markers of synaptic plasticity associated with hippocampal memory formation, we did observe elevated expression of Glut4 and AdipoR1 (Fig 6b) suggesting enhanced glucose transport and adiponectin signaling in the brain. This novel finding that Fisetin treatment concurrently improved peripheral and central glucose, and adiponectin signaling has important implications for metabolic and cognitive function. In the periphery, adiponectin increases insulin sensitivity, stimulates fatty acid oxidation and glucose uptake, suppresses hepatic glucose production ${ }^{30}$, and inhibits inflammation ${ }^{31}$. In the brain, adiponectin signaling via AdipoR1 has anti-inflammatory effects and is involved with cognitive and neuroprotective mechanisms ${ }^{32}$. Monthly Fisetin treatment in male mice led to increased adiponectin levels in circulation, which likely contributed to improvements of peripheral glucose and lipid metabolism. Circulating adiponectin passes through the blood brain barrier where it can enhance cognitive function by upregulating glucose transport and AdipoR1 signaling ${ }^{32}$. However, neither 
Glut4, AdipoR1, nor the examined synaptic plasticity markers (Figs. 6c and 6d) were affected by $\mathrm{D}+\mathrm{Q}$ treatment in the female mice even though object memory retention was reduced (Fig. 5f). The reduced cognitive performance of these animals might be attributed to the increased adiposity after D+Q treatment, but future studies are needed to identify these mechanisms involved.

Cell senescence is strongly correlated with aging, but currently no diagnostic testing is available to quantify senescent cells accumulation. It seems possible that the earlier in life senotherapeutics are taken, the better likelihood of delaying age-related decline and disease risk might be expected. Senolytics such as Fisetin and D+Q are known to reduce senescent cell burden ${ }^{1}$, but the optimal age to begin treatment is currently unknown. Our study indicates that both age and sex may also determine the therapeutic outcomes of senolytic treatment. When the treatment was started at 4 months of age, before the reported senescent cell accumulation, Fisetin had beneficial effects in male mice while a D+Q cocktail had adverse consequences in female mice. These observations provide novel information with translational relevance. First, senolytic drugs can be taken at an age before significant senescent cell burden to reduce or prevent their prevalence later in life. Second, males and females have differential responses to the same senolytic treatment when initiated at younger ages. Third, a particular senolytic treatment may have beneficial, negligible or detrimental effects depending on the age, sex, or disease. These observations should serve as a note of caution in this rapidly evolving and expanding field of investigation. Fourth, oral senolytic treatment not only impacted peripheral physiological responses, but also affected cognitive performance, possibly via increased central adiponectin signaling and glucose transport. 
Particularly striking, these effects were observed during an age when age-related cognitive decline is not typically apparent in C57BL/6 mice.

Designing senotherapies with the role of sex and age of onset could conceivably provide a useful preventative treatment strategy for dealing with development of neurodegenerative disorders. Supporting this possibility, development of metabolic syndrome in midlife was reported to increases the risk for Alzheimer's disease (AD) later on 33. Besides clearance of senescent cells, senotherapeutics may ameliorate or delay cognitive decline through mechanisms associated with improved peripheral and central glucose metabolism. Considering metabolic dysregulation has been observed in the APP/PS1 model of $\mathrm{AD}^{34}$, Additional studies are warranted to determine if prodromal senolytic treatment could provide cognitive benefits in neurodegenerative diseases. 


\section{Material and methods}

\section{Chemicals}

Unless otherwise noted, all chemicals were obtained from Sigma-Aldrich (St. Louis, MO) including Quercetin (Cat\# RHR1488). Fisetin was purchased from Selleckchem (Houston, TX; Cat \#S2298), and Dasatinib from LC laboratories (Woburn, MA; Cat\# D-3307).

\section{Animals}

Male and female C57BL/6 mice were maintained in our established breeding colonies. Protocols for animal use were approved by the Institutional Animal Care and Use Committee at Southern Illinois University School of Medicine. Mice were group housed on a 12:12 h light-dark cycle, and food (Chow 5001 with 23.4\% protein, 5\% fat, and 5.8\% crude fiber; LabDiet PMI Feeds) and water were available ad libitum. As shown in Fig 1a, glucose metabolism was accessed via ITT and GTT after 6 treatments at the animal age of 9 months. NOR and MWM was performed after nine treatments at the age of 12 months. Energy metabolism was measured by indirect calorimetry at the animal age of 12.5 to 13 months. Afterwards, mice were euthanized with an overdose of isoflurane followed by rapid decapitation and plasma and tissues were collected as previously described ${ }^{35}$.

\section{Senolytic drug treatment}

The senolytic drug treatments were based on previous publications ${ }^{17,36}$. C57BL/6 mice were dosed with $100 \mathrm{mg} / \mathrm{kg}$ of Fisetin while a cocktail of $5 \mathrm{mg} / \mathrm{kg}$ of D+ $50 \mathrm{mg} / \mathrm{kg}$ of Q, or vehicle (2\% DMSO in canola oil) by oral administration ${ }^{37}$. The treatments were given once per month from 4-13 months of age. 


\section{Glucose tolerance test (GTT) and insulin tolerance test (ITT)}

GTT or ITT was carried out as described previously ${ }^{35}$. Sixteen-hour-fasted mice underwent GTT by intraperitoneal (i.p.) injection with $1 \mathrm{~g}$ glucose per $\mathrm{kg}$ of body weight (BW). Blood glucose levels were measured at 0, 15, 30, 45, 60, and 120 min with a PRESTO glucometer (AgaMatrix). For ITT, nonfasted mice were injected i.p. with 1 IU porcine insulin from sigma (St. Louis, MO) (Cat\# I5523) per kg of BW. Blood glucose levels were measured at 0, 15, 30, 45, 60, and 120 min. The data for GTT are presented as absolute value, and for ITT are presented as a percentage of baseline glucose.

\section{Indirect calorimetry}

Indirect calorimetry was performed as previously described ${ }^{35}$ using AccuScan Metabolic System (AccuScan Instruments). In this system, mice are housed individually in metabolic chambers with ad libitum access to food and water. After a 24-hr acclimation period, $\mathrm{VO}_{2}, \mathrm{VCO}_{2}$, EE and RQ measurements were collected every $10 \mathrm{~min}$. Gas samples were collected and analyzed every 10 min per animal, and the data were averaged for each hour.

\section{Morris water maze (MWM) training and probe challenge}

The MWM was used to assess spatial learning and memory recall, and performed as previously described ${ }^{38}$. Mice were trained to utilize visual cues placed around the room to repeatedly swim to a static, hidden escape platform (submerged $1 \mathrm{~cm}$ below the opaque water surface), regardless of starting quadrant. The MWM paradigm consisted of 5 consecutive training days with three, 90 
$\mathrm{s}$ trials/day and a minimum inter-trial-interval of $20 \mathrm{~min}$. Starting quadrant was randomized for each trial. After two days without testing, the escape platform was removed and all mice entered the pool of water from the same starting position for a single $60 \mathrm{~s}$ probe challenge to test long-term memory recall. The ANY-maze video tracking system (Stoelting Co., Wood Dale, IL) was used to record mouse navigation during the training and probe challenge. The three trials for each training day were averaged for each mouse.

\section{Novel Object Recognition (NOR)}

The NOR was used to evaluate memory retention based on the premise that mice spend more time exploring a novel object rather than a familiar object if memory capabilities remain intact. Mice were habituated in the open field chamber for $30 \mathrm{~min}$ on the first day. Twenty-four hours after, the mouse was returned to the chamber and presented with two similar objects for 5 min. A $24 \mathrm{hr}$ inter-session-interval was used between introduction and retention phases to assess longterm memory retrieval. During the retention phase one of the familiar objects is replaced with a novel object and the mouse is given 5 minutes of exploration. The ANY-maze video tracking system (Stoelting Co., Wood Dale, IL) was used to record mouse navigation during the familiarization and retention phases and used to assay the retention index.

\section{Assessment of blood chemistry}

Plasma was collected from animals anesthetized with isoflurane by cardiac puncture at sacrifice. The blood was mixed with EDTA, followed by centrifugation at $10,000 \mathrm{~g}$ for $15 \mathrm{~min}$ at $4^{\circ} \mathrm{C}$ for plasma collection. Per the manufacturer's protocol, insulin or adiponectin was measured with 
respective ELISA kits that were purchased from Crystal Chem (Elk Grove Village, IL; Cat\# 90080 and 80569).

\section{RT-PCR}

mRNA expression was analyzed by quantitative RT-PCR as previously described ${ }^{35}$ using cDNA synthesis kits and SYBR green mix from Bio-Rad (Cat\# 1708897 and 1725121) performed with the StepOne Real-Time PCR System (Thermo Fisher Scientific). RNA was extracted using an RNeasy mini kit or RNeasy Lipid Tissue Mini Kit (Qiagen) following the manufacturer's instructions. Relative expression was calculated as previously described (Masternak et al., 2012) and primers were purchased from Integrated DNA Technologies (Table 1).

\section{Statistical analysis}

Differences between two groups were assessed with unpaired two-tailed Student's t tests with significance defined as $\mathrm{p}<0.05$. Data are presented as mean \pm SEM. All statistical analyses and graphs were completed using Prism 6 (GraphPad Inc, La Jolla, CA, USA). 


\section{Acknowledgements}

This work was supported by the National Institutes of Health NIA R01-AG057767 and NIA R01AG061937, Dale and Deborah Smith Center for Alzheimer's Research and Treatment, Kenneth Stark Endowment (YF, SF, KQ, MRP, KNH, ERH), NIA R21-AG062985, and American Diabetes Association 1-19-IBS-126 (DM, RS, AB). We would like to thank Lisa Hensley for editorial support.

\section{Author Contributions}

YF, DM, RS, SM, KQ, and MRP conducted the experiments and analyzed the data. YF, AB, KNH, and ERH conceived the study, designed the experiments, interpreted the data, and wrote the manuscript. All authors approved the final version of the manuscript.

\section{Conflict of Interest}

The authors declare no conflicts of interest. 


\section{References}

$1 \quad$ Kirkland, J. L. \& Tchkonia, T. Senolytic drugs: from discovery to translation. J Intern Med 288, 518536, doi:10.1111/joim.13141 (2020).

2 Campisi, J. Senescent cells, tumor suppression, and organismal aging: good citizens, bad neighbors. Cell 120, 513-522, doi:10.1016/j.cell.2005.02.003 (2005).

3 Lasry, A. \& Ben-Neriah, Y. Senescence-associated inflammatory responses: aging and cancer perspectives. Trends Immunol 36, 217-228, doi:10.1016/j.it.2015.02.009 (2015).

4 Freund, A., Orjalo, A. V., Desprez, P. Y. \& Campisi, J. Inflammatory networks during cellular senescence: causes and consequences. Trends Mol Med 16, 238-246, doi:10.1016/j.molmed.2010.03.003 (2010).

5 McHugh, D. \& Gil, J. Senescence and aging: Causes, consequences, and therapeutic avenues. J Cell Biol 217, 65-77, doi:10.1083/jcb.201708092 (2018).

6 Chen, J. H., Hales, C. N. \& Ozanne, S. E. DNA damage, cellular senescence and organismal ageing: causal or correlative? Nucleic Acids Res 35, 7417-7428, doi:10.1093/nar/gkm681 (2007).

7 Chapman, J., Fielder, E. \& Passos, J. F. Mitochondrial dysfunction and cell senescence: deciphering a complex relationship. FEBS Lett 593, 1566-1579, doi:10.1002/1873-3468.13498 (2019).

8 Prata, L., Ovsyannikova, I. G., Tchkonia, T. \& Kirkland, J. L. Senescent cell clearance by the immune system: Emerging therapeutic opportunities. Semin Immunol 40, 101275, doi:10.1016/j.smim.2019.04.003 (2018).

9 Passos, J. F., Miwa, S. \& von Zglinicki, T. Measuring reactive oxygen species in senescent cells. Methods Mol Biol 965, 253-263, doi:10.1007/978-1-62703-239-1_17 (2013).

10 Musi, N. et al. Tau protein aggregation is associated with cellular senescence in the brain. Aging Cell 17, e12840, doi:10.1111/acel.12840 (2018).

11 Ogrodnik, M. et al. Obesity-Induced Cellular Senescence Drives Anxiety and Impairs Neurogenesis. Cell Metab 29, 1233, doi:10.1016/j.cmet.2019.01.013 (2019).

12 Grynkiewicz, G. \& Demchuk, O. M. New Perspectives for Fisetin. Front Chem 7, 697, doi:10.3389/fchem.2019.00697 (2019).

13 Jafarinia, M. et al. Quercetin with the potential effect on allergic diseases. Allergy Asthma Clin Immunol 16, 36, doi:10.1186/s13223-020-00434-0 (2020).

14 Mlcek, J., Jurikova, T., Skrovankova, S. \& Sochor, J. Quercetin and Its Anti-Allergic Immune Response. Molecules 21, doi:10.3390/molecules21050623 (2016).

15 Aguilera, D. G. \& Tsimberidou, A. M. Dasatinib in chronic myeloid leukemia: a review. Ther Clin Risk Manag 5, 281-289, doi:10.2147/tcrm.s3425 (2009).

16 Zhu, Y. et al. The Achilles' heel of senescent cells: from transcriptome to senolytic drugs. Aging Cell 14, 644-658, doi:10.1111/acel.12344 (2015).

17 Yousefzadeh, M. J. et al. Fisetin is a senotherapeutic that extends health and lifespan. EBioMedicine 36, 18-28, doi:10.1016/j.ebiom.2018.09.015 (2018).

$18 \mathrm{Xu}, \mathrm{M}$. et al. Senolytics improve physical function and increase lifespan in old age. Nat Med 24, 1246-1256, doi:10.1038/s41591-018-0092-9 (2018).

19 Baker, D. J. et al. Clearance of p16Ink4a-positive senescent cells delays ageing-associated disorders. Nature 479, 232-236, doi:10.1038/nature10600 (2011).

20 Bussian, T. J. et al. Clearance of senescent glial cells prevents tau-dependent pathology and cognitive decline. Nature 562, 578-582, doi:10.1038/s41586-018-0543-y (2018).

21 Zhang, P. et al. Senolytic therapy alleviates Abeta-associated oligodendrocyte progenitor cell senescence and cognitive deficits in an Alzheimer's disease model. Nat Neurosci 22, 719-728, doi:10.1038/s41593-019-0372-9 (2019). 

Suppression of IL-1R/TLR Axis and Apoptosis in Pentylenetetrazole-Induced Kindling in Mice. Front Neurol 12, 689069, doi:10.3389/fneur.2021.689069 (2021).

23 Cannon, B. \& Nedergaard, J. Brown adipose tissue: function and physiological significance. Physiol Rev 84, 277-359, doi:10.1152/physrev.00015.2003 (2004).

24 Kalinovich, A. V., de Jong, J. M., Cannon, B. \& Nedergaard, J. UCP1 in adipose tissues: two steps to full browning. Biochimie 134, 127-137, doi:10.1016/j.biochi.2017.01.007 (2017).

25 Azzu, V. \& Valencak, T. G. Energy Metabolism and Ageing in the Mouse: A Mini-Review. Gerontology 63, 327-336, doi:10.1159/000454924 (2017).

26 Westbrook, R., Bonkowski, M. S., Strader, A. D. \& Bartke, A. Alterations in oxygen consumption, respiratory quotient, and heat production in long-lived GHRKO and Ames dwarf mice, and shortlived bGH transgenic mice. J Gerontol A Biol Sci Med Sci 64, 443-451, doi:10.1093/gerona/gln075 (2009).

27 Prentice, R. L. et al. An exploratory study of respiratory quotient calibration and association with postmenopausal breast cancer. Cancer Epidemiol Biomarkers Prev 22, 2374-2383, doi:10.1158/1055-9965.EPI-13-0511 (2013).

28 Reshetnikov, V. V. et al. Genes associated with cognitive performance in the Morris water maze: an RNA-seq study. Sci Rep 10, 22078, doi:10.1038/s41598-020-78997-6 (2020).

29 Tchkonia, T. et al. Fat tissue, aging, and cellular senescence. Aging Cell 9, 667-684, doi:10.1111/j.1474-9726.2010.00608.x (2010).

30 Roy, B. \& Palaniyandi, S. S. Tissue-specific role and associated downstream signaling pathways of adiponectin. Cell Biosci 11, 77, doi:10.1186/s13578-021-00587-4 (2021).

31 Bloemer, J. et al. Role of Adiponectin in Central Nervous System Disorders. Neural Plast 2018, 4593530, doi:10.1155/2018/4593530 (2018).

32 Rastegar, S. et al. Expression of adiponectin receptors in the brain of adult zebrafish and mouse: Links with neurogenic niches and brain repair. J Comp Neurol 527, 2317-2333, doi:10.1002/cne.24669 (2019).

33 Baumgart, M. et al. Summary of the evidence on modifiable risk factors for cognitive decline and dementia: A population-based perspective. Alzheimers Dement 11, 718-726, doi:10.1016/j.jalz.2015.05.016 (2015).

34 Hascup, E. R. et al. Diet-induced insulin resistance elevates hippocampal glutamate as well as VGLUT1 and GFAP expression in AbetaPP/PS1 mice. J Neurochem 148, 219-237, doi:10.1111/jnc.14634 (2019).

35 Fang, Y. et al. Lifespan of long-lived growth hormone receptor knockout mice was not normalized by housing at 30 degrees C since weaning. Aging Cell 19, e13123, doi:10.1111/acel.13123 (2020).

36 Palmer, A. K. et al. Cellular Senescence in Type 2 Diabetes: A Therapeutic Opportunity. Diabetes 64, 2289-2298, doi:10.2337/db14-1820 (2015).

37 Dalterio, S. \& Bartke, A. Perinatal exposure to cannabinoids alters male reproductive function in mice. Science 205, 1420-1422, doi:10.1126/science.472762 (1979).

38 Hascup, K. N., Findley, C. A., Sime, L. N. \& Hascup, E. R. Hippocampal alterations in glutamatergic signaling during amyloid progression in AbetaPP/PS1 mice. Sci Rep 10, 14503, doi:10.1038/s41598-020-71587-6 (2020). 


\section{Figure legends}

Figure 1: Senolytic treatment altered the SASP profile in C57BL/6 mice. (a) Experimental paradigm. (b-g) Gene expressions in gonadal fat depot. Data are represented as means $\pm \operatorname{SEM}(n$ $=8-12) . * p \leq 0.05, * * p \leq 0.01, * * * \mathrm{p} \leq 0.001$ based on a two-tailed Student's $t$ test.

Figure 2: $D+Q$ treatment increased abdominal WAT by upregulation of lipid synthesis and downregulation of thermogenesis in female mice. Mouse body weight (a, BW) and percentage of total (b), subQ (c), gonadal (d), perirenal (e) and interscapular brown (f) adipose tissue present in relation to BW. Gene expression in gonadal fat $(\mathrm{g})$ and interscapular brown fat $(\mathrm{h}-\mathrm{i})$ depots. Data are means \pm SEM $(n=8-20) . * p \leq 0.05, * * p \leq 0.01$ based on a two-tailed Student's t test.

Figure 3: Fisetin treatment improved glucose metabolism and increased plasma adiponectin levels in the male mice. Glucose tolerance test (a-b; GTT) and area under the curve (c; AUC) after five treatments. Insulin tolerance test (d-e; ITT) and AUC (f) after five treatments. Plasma insulin (g) and adiponectin (h) levels taken at time of euthanization after 9 treatments. Data are means \pm SEM $(n=16-20) .{ }^{*} \mathrm{p} \leq .05$ based on a two-tailed Student's $t$ test.

Figure 4: Fisetin treatment enhanced energy metabolism in male mice, while $D+Q$ treatment decreased energy metabolism in female mice. Twenty-four hour male and female oxygen consumption ( $\mathrm{a}-\mathrm{b} ; \mathrm{VO}_{2}$ ), energy expenditure (c-d; EE), and respiratory quotient (e-f; RQ) along with their corresponding AUC after 9 treatments. Data are presented as means $\pm \operatorname{SEM}(\mathrm{n}=$ $16-20) .{ }^{* *} \mathrm{p} \leq 0.01$ based on a two-tailed Student's t test.

Figure 5: Fisetin treatment improved spatial learning in male mice, while $D+Q$ treatment impaired object recognition memory recall in female mice. Assessment of cognition and memory recall was determined by MWM and NOR after 9 treatments. MWM Path efficiency and corrected integrated path length (CIPL) along with their corresponding area under the curve in male ( $\mathrm{a} \& \mathrm{~b}$ ) and female (c \& d) C57BL/6 mice during 5 training sessions. Number of platform entries during the delayed probe challenge (e). Retention index of the familiar object after a delayed novelty introduction (f). Data are presented as means $\pm \operatorname{SEM}(n=16-20) .{ }^{*} p \leq$ $0.05, * * \mathrm{p} \leq 0.01$ based on a two-tailed Student's t test.

Figure 6: Fisetin treatment enhanced expression of Glut4 and AdipoR1 in male mice. Gene expression in hippocampus levels were measured at time of euthanization after 10 treatments (ad). Data are presented as means $\pm \operatorname{SEM}(n=16-20)$. ${ }^{*} \mathrm{p} \leq 0.05, * * \mathrm{p} \leq 0.01$ based on a two-tailed Student's t test.

Table 1: A list of forward and reverse primers used in this study. 


\section{Figure 1}

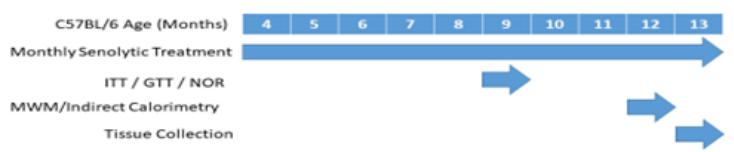

Fig. 1a
4 months (non-treated)

21 months (non-treated)

Con 13 months

$D+Q 13$ months

E Fisetin 13 months

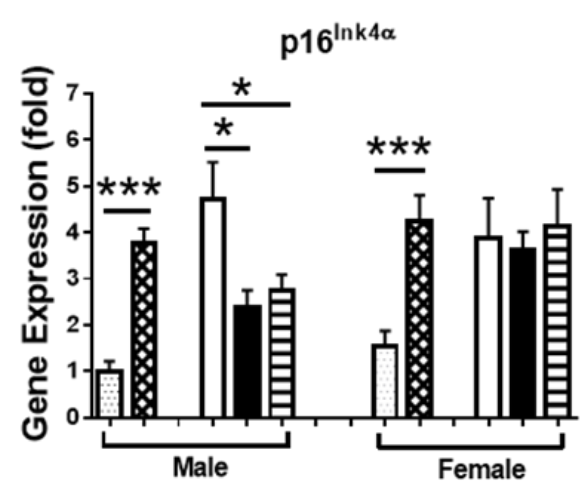

Fig. 1b

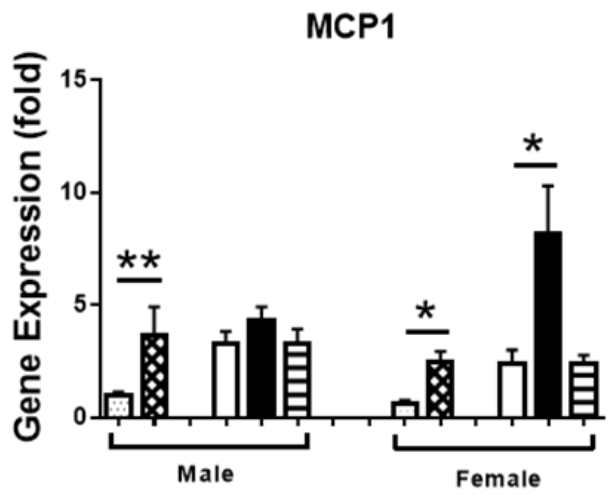

Fig. 1e p21 ${ }^{\text {cip1 }}$

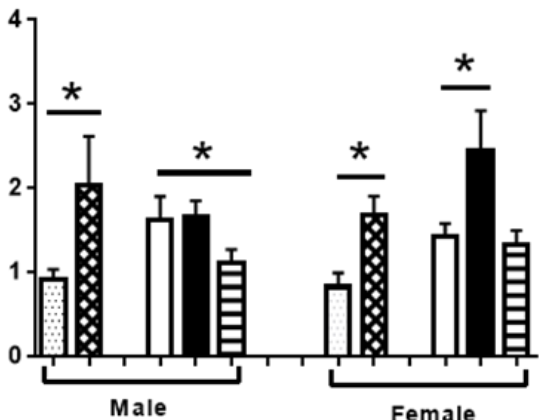

Fig. 1c

IL-6

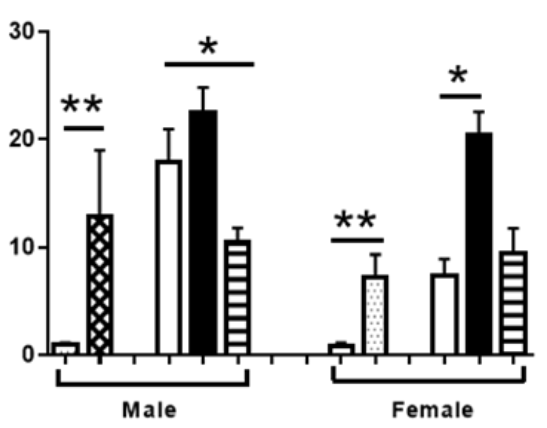

Fig. 1f
TNF $\alpha$

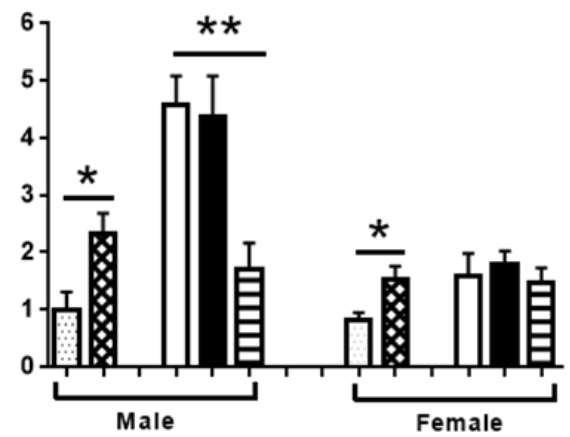

Fig. 1d

IL-10



Fig. 1g 
Figure 2

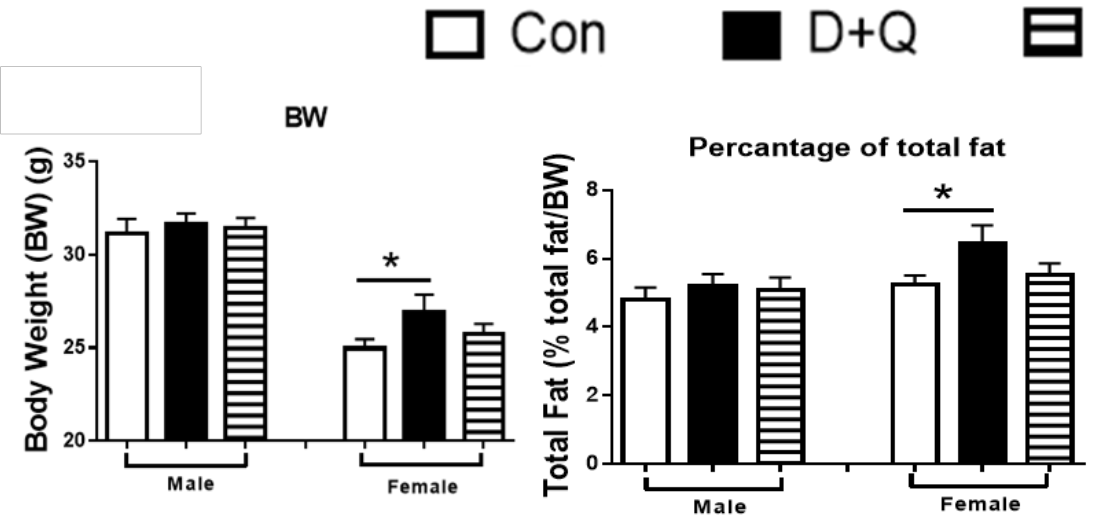

Fig. 2a

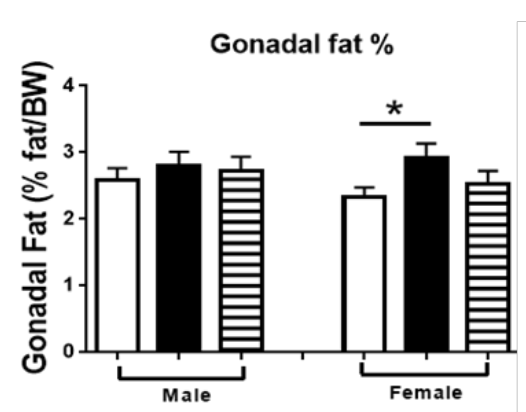

Fig. 2d
Fig. 2b

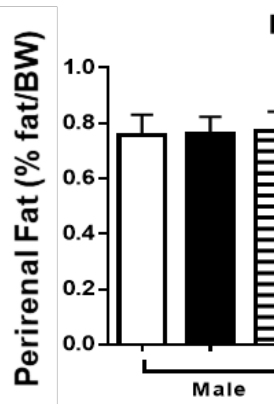

Peri. Fat $\%$

Fig. 2e
Fisetin

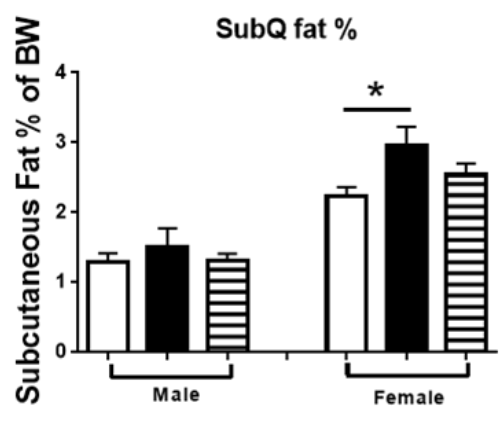

Fig. 2c

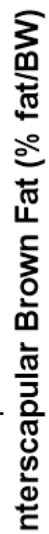



Interscapular BAT \%

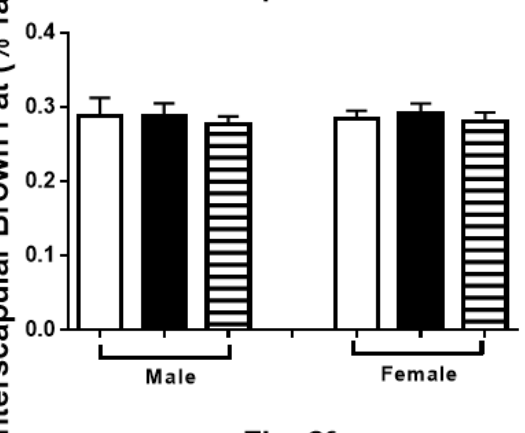

Fig. $2 f$

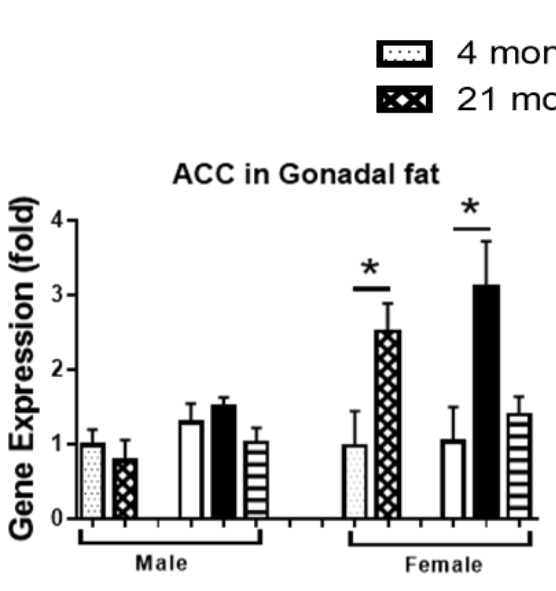

Fig. 2g
UCP1 in interscapular BAT

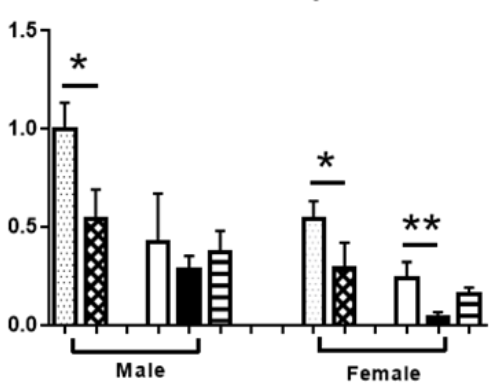

Fig. $2 \mathrm{~h}$

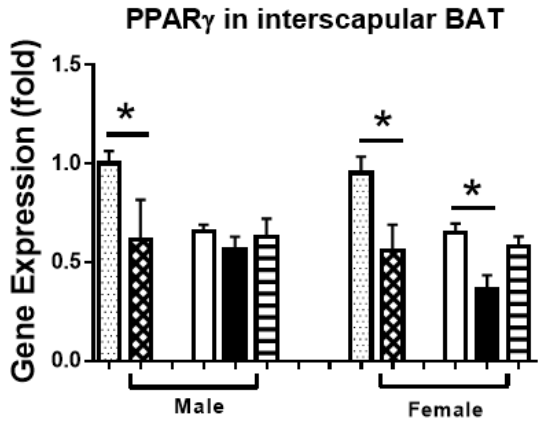

Fig. 2i 


\section{Figure 3}

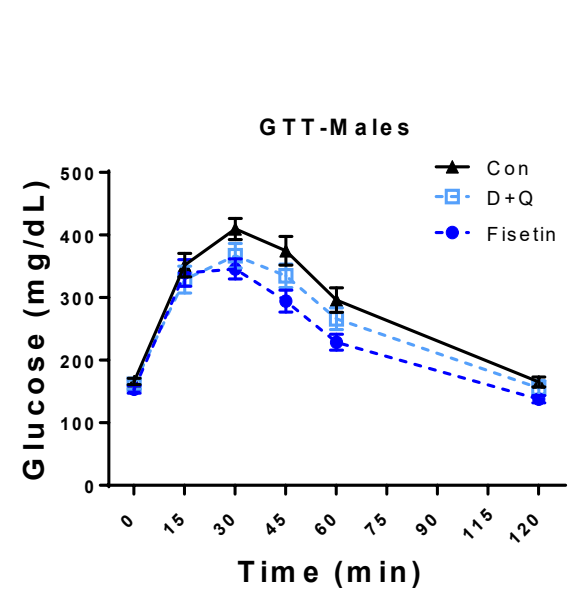

Fig. $3 a$

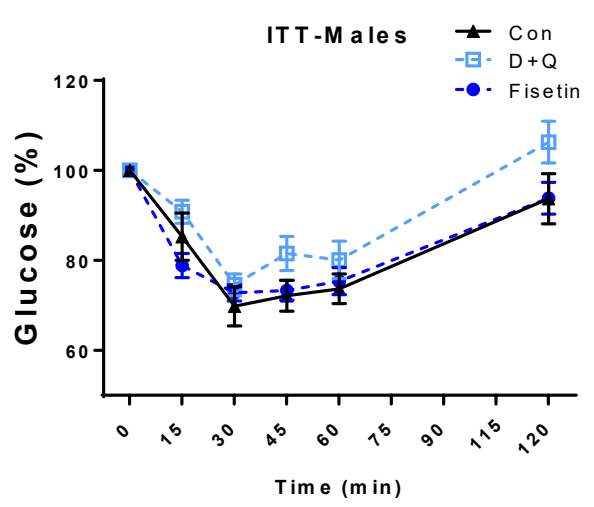

Fig. 3d

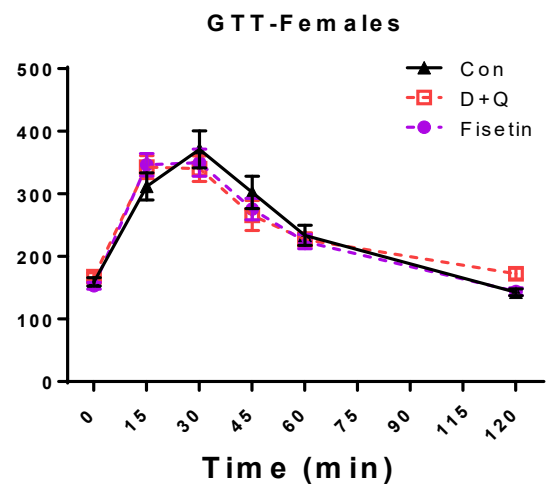

Fig. $3 b$

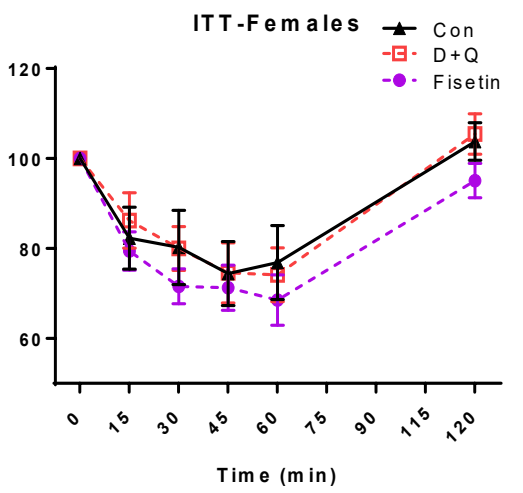

Fig. 3e $\square$ Con $\square \mathrm{D}+\mathrm{Q}$ 日 Fisetin

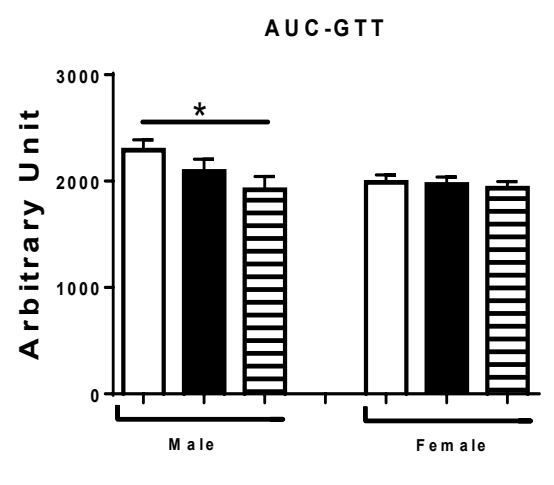

Fig. 3c

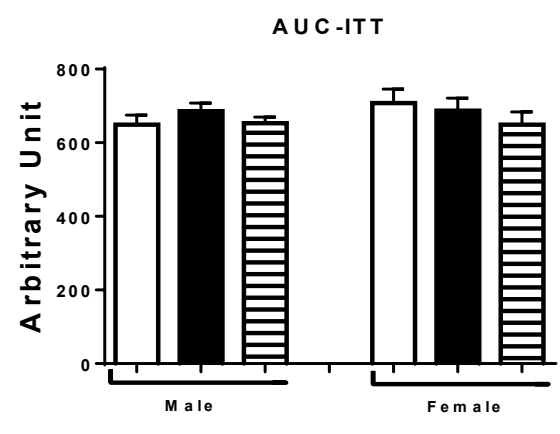

Fig. $3 f$
Insulin in plasma

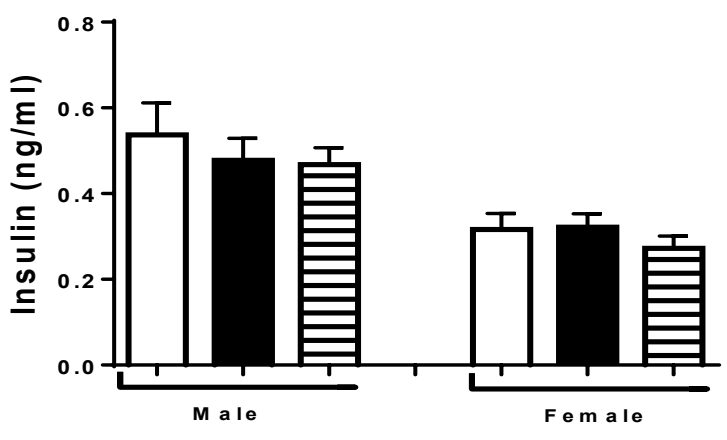

Fig. $\mathbf{3 g}$
Adiponectin in plasma

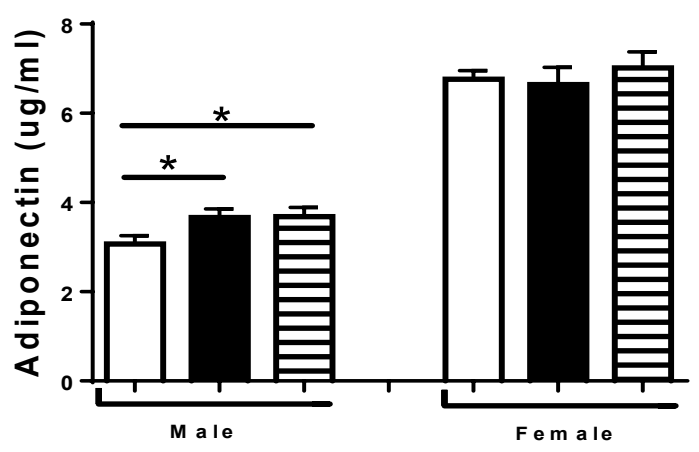

Fig . $3 \mathrm{~h}$ 
Figure 4

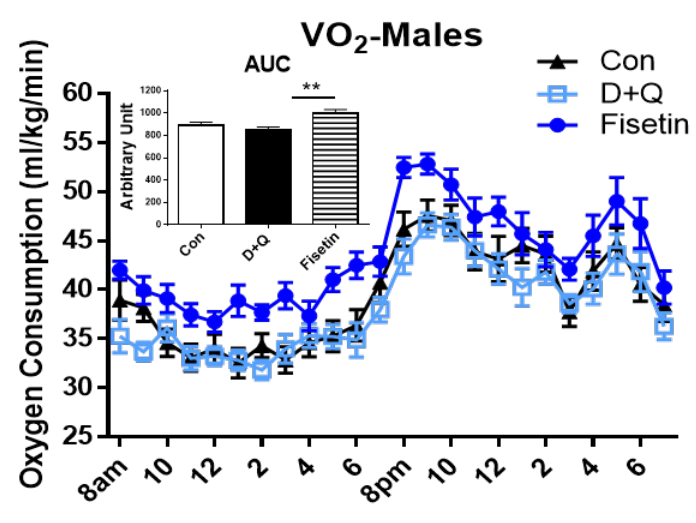

Time

Fig. 4a

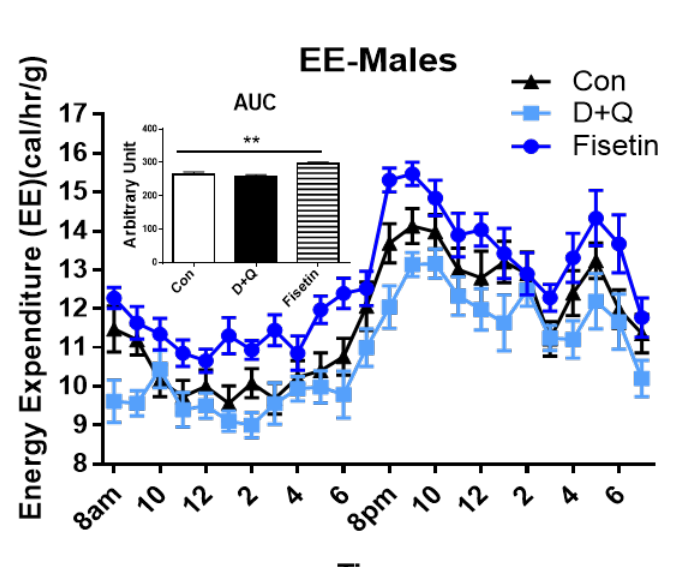

Fig. 4c

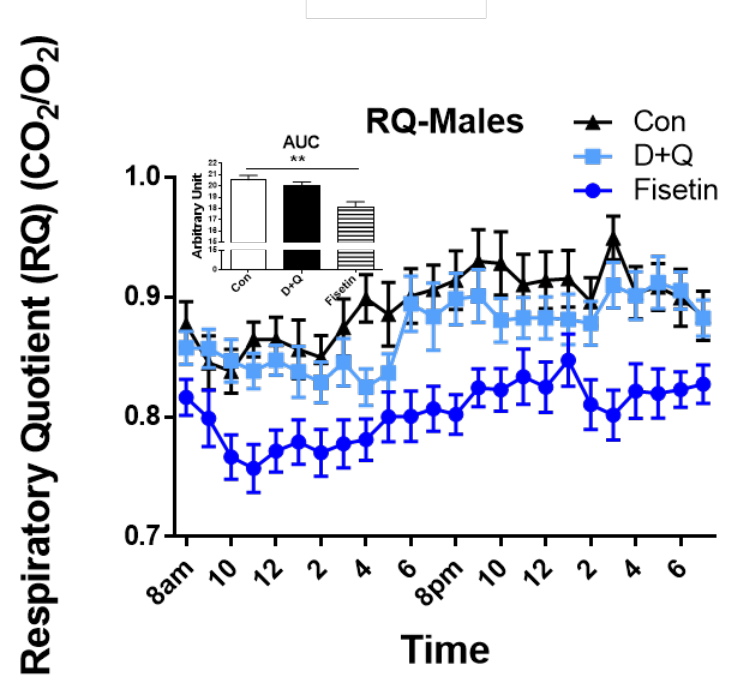

Fig. 4e

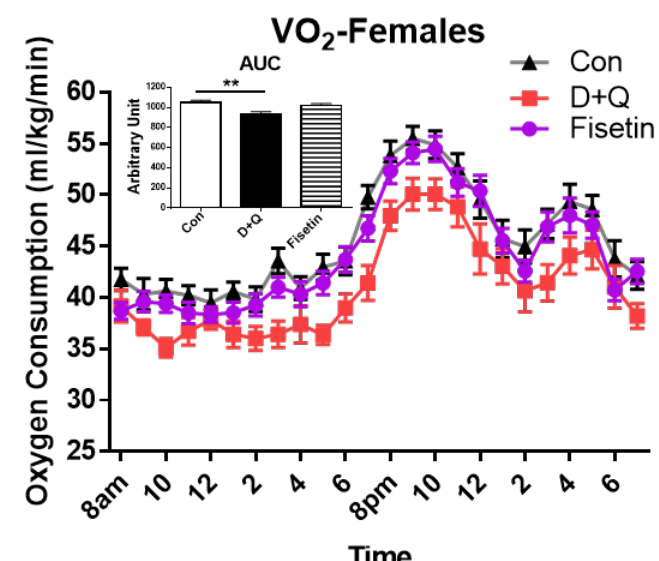

Fig. 4b

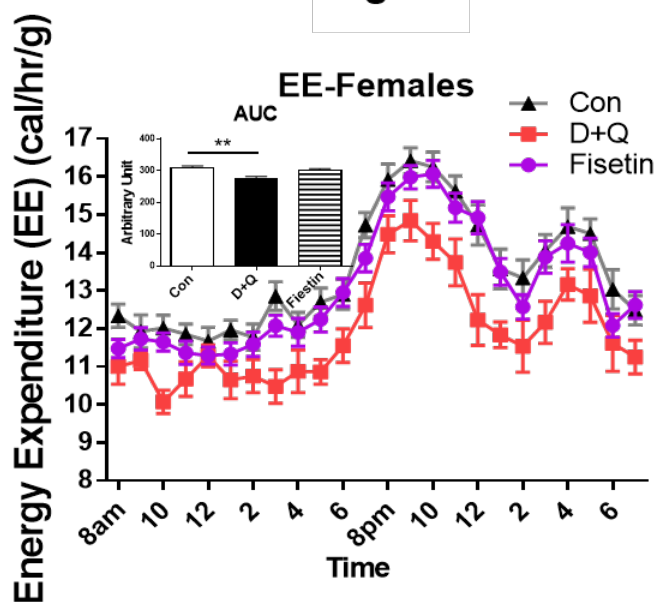

Fig. 4d

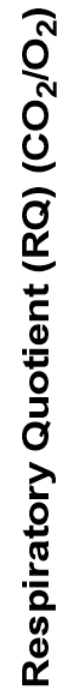

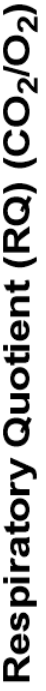

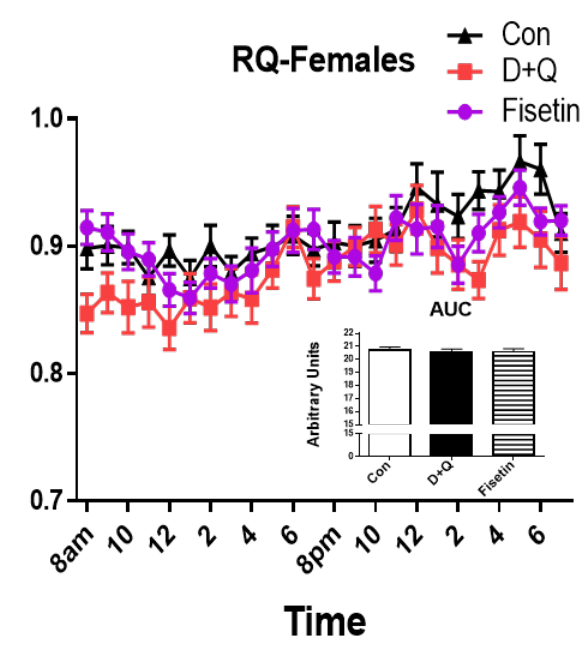

Fig. $4 f$ 


\section{Figure 5}

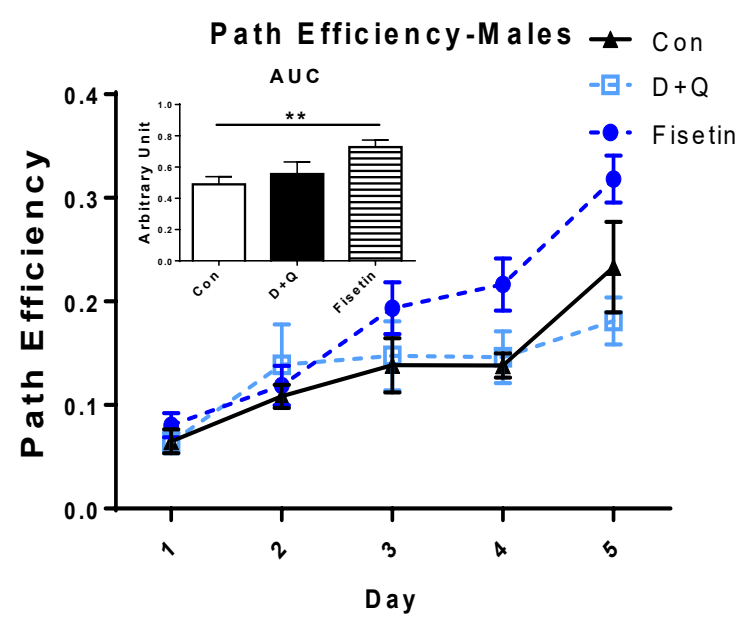

Fig. 5 a

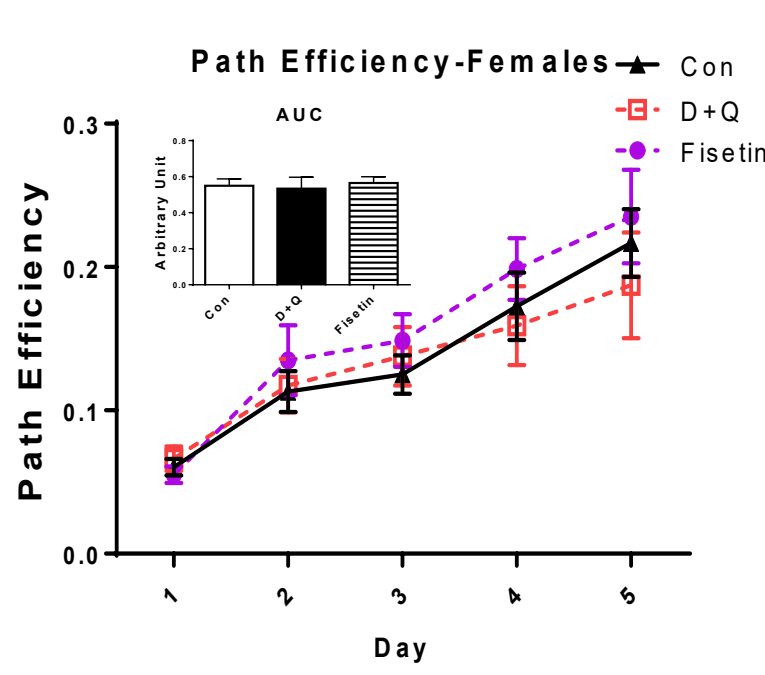

Fig. 5c

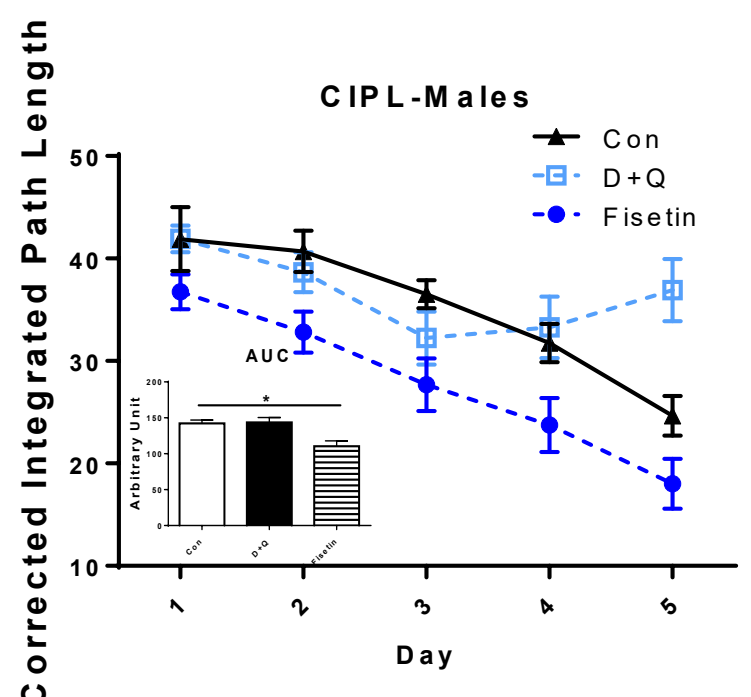

Fig. 5b

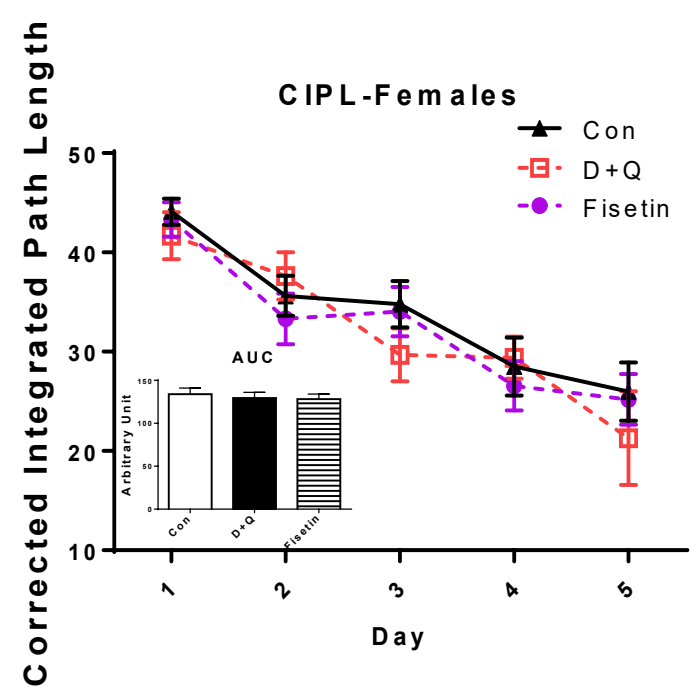

Fig. 5d

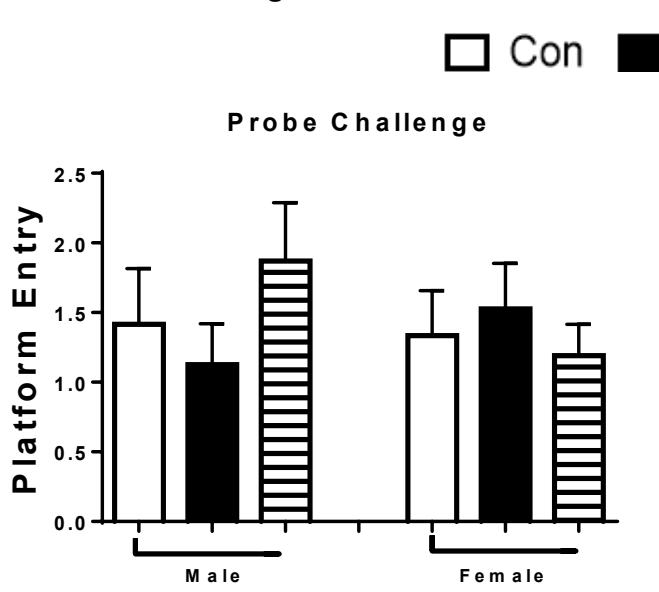

Fig. 5e

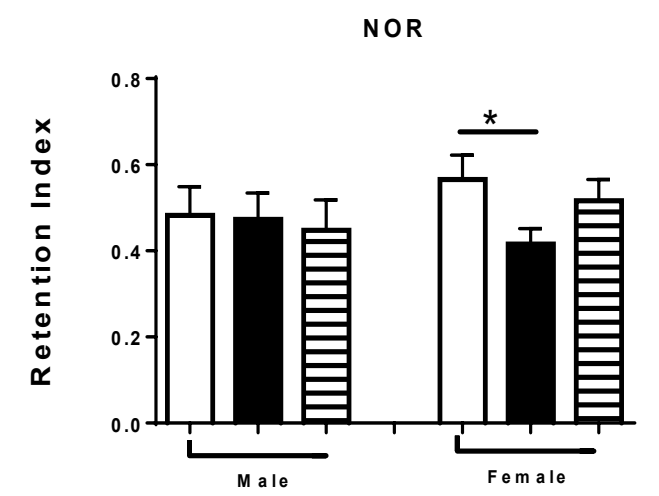

Fig. $5 f$ 
Figure 6
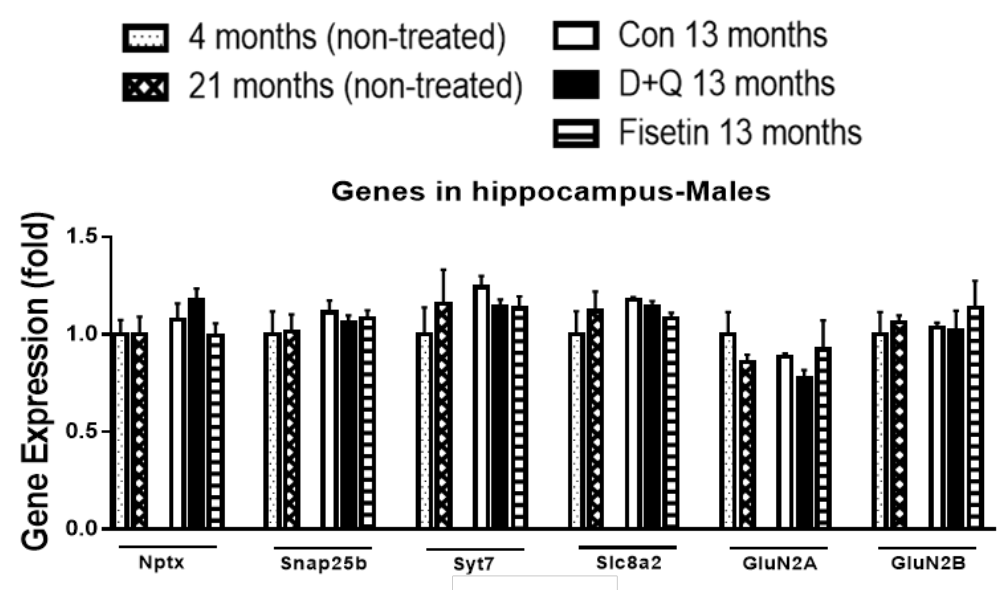

Fig. 6a

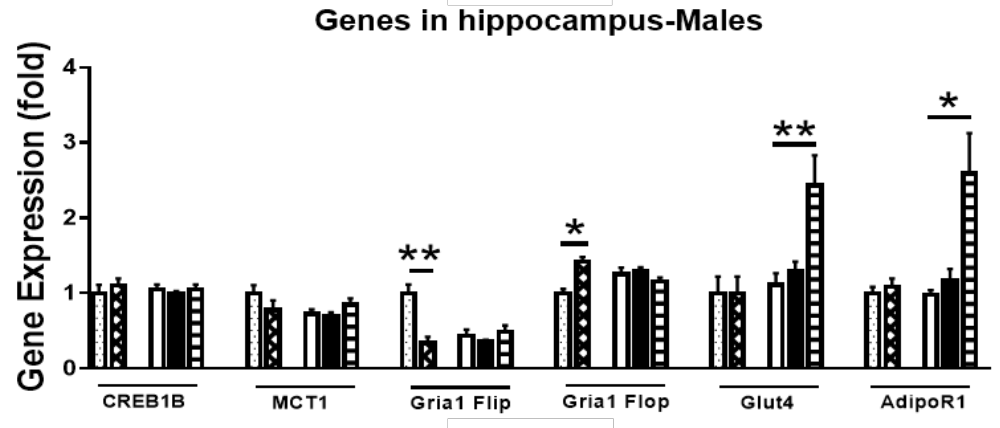

Fig. 6b

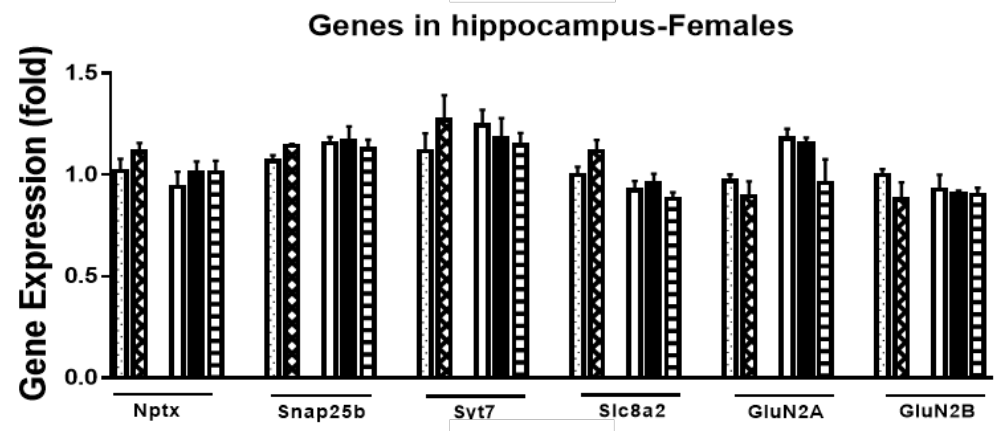

Fig. 6c

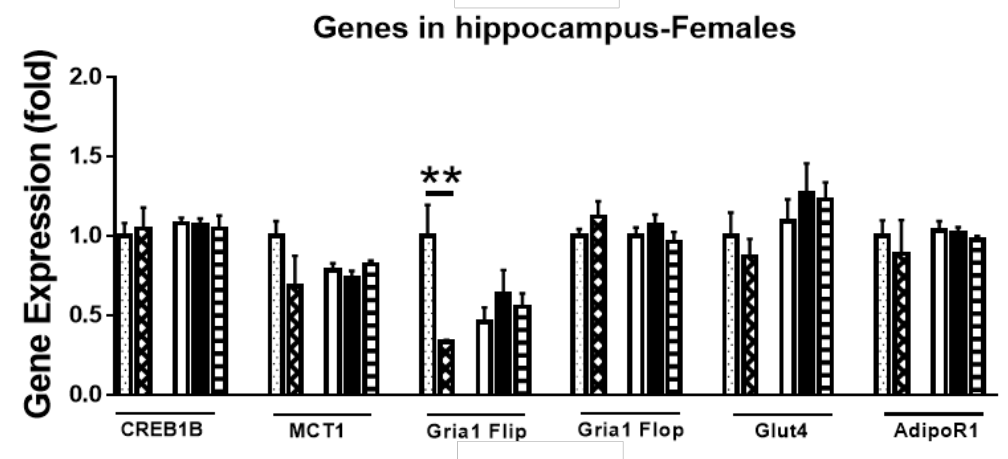

Fig. 6d 
Table 1

\begin{tabular}{|c|c|c|c|}
\hline Gene & Protein & Forward & $\underline{\text { Reverse }}$ \\
\hline CDKN2A & $\mathrm{p} 16^{\text {Ink4a }}$ & TACCCCGATTCAGGTGAT & TTGAGCAGAAGAGCTGCTACGT \\
\hline CDKN1A & $\mathrm{p} 21^{\mathrm{Cip} 1}$ & CCTGGTGATGTCCGACCTG & CCATGAGCGCATCGCAATC \\
\hline $\mathrm{TNF} \alpha$ & $\mathrm{TNF} \alpha$ & GCCTCTTCTCATTCCTGCTTG & CTGATGAGAGGGAGGCCATT \\
\hline CCL2 & MCP1 & CCACTCACCTGCTGCTACTCAT & TGGTGATCCTCTTGTAGCTCTCC \\
\hline IL6 & IL-6 & TAGTCCTTCCTACCCCAATTTCC & TTGGTCCTTAGCCACTCCTTC \\
\hline IL10 & IL-10 & GCTCTTACTGACTGGCATGAG & CGCAGCTCTAGGAGCATGTG \\
\hline ACACA & $\mathrm{ACC}$ & ATGGGCGGAATGGTCTCTTTC & TGGGGACCTTGTCTTCATCAT \\
\hline UCP1 & UCP1 & AGGCTTCCAGTACCATTAGGT & CTGAGTGAGGCAAAGCTGATTT \\
\hline PPARG & $\operatorname{PPAR} \gamma$ & ACCCCCTGCTCCAGGAGAT & TGCAATCAATAGAAGGAACACGT \\
\hline NPTX & Nptx & ACACCATGAAAGTGGGAGGTAACT & ACTGGTCTTGTCCTTACTTCCGGA \\
\hline SNAP25 & Snap25b & CTCATCGAGTGGGGCAACA & GTGATGCCATTTGCCATCGTT \\
\hline Syt7 & Syt7 & ACTCCATCATCGTGAACATCATC & TATGTCGAAGGCGAAAGAC \\
\hline Slc8a2 & $\mathrm{Na}^{+} / \mathrm{Ca}^{++}$Exchanger, Member 2 & AACAGCACCTTCTACGTGG & GTCCTTCTGCGTCTCCC \\
\hline GRIN2A & GluN2A & ACGTGACAGAACGCGAACTT & TCAGTGCGGTTCATCAATAACG \\
\hline GRIN2B & GluN2B & CAGCAAAGCTCGTTCCCAAAA & GTCAGTCTCGTTCATGGCTAC \\
\hline CREB1 & Creb1b & AGCAGCTCATGCAACATCATC & AGTCCTTACAGGAAGACTGAACT \\
\hline SLC16A1 & MCT1 & TGTTAGTCGGAGCCTTCATTTC & CACTGGTCGTTGCACTGAATA \\
\hline GRIA1 & Gria1 Flip & ACACCATGAAAGTGGGAGGTAACT & ACTGGTCTTGTCCTTACTTCCGGA \\
\hline GRIA1 & Gria1 Flop & GTCCGCCCTGAGAAATCCA & GCACTCGCCCTTGTCGTA \\
\hline SLC2A4 & Glut4 & ACACTGGTCCTAGCTGTATTCT & CCAGCCACGTTGCATTGTA \\
\hline ADIPOR1 & AdipoR1 & ACGTTGGAGAGTCATCCCGTAT & CTCTGTGTGGATGCGGAAGAT \\
\hline $\mathrm{B} 2 \mathrm{M}$ & $\mathrm{B} 2 \mathrm{M}$ & TTCTGGTGCTTGTCTCACTGA & CAGTATGTTCGGCTTCCCATTC \\
\hline
\end{tabular}

